\title{
Cephalic lateral crural advancement flap
}

\author{
Fuat Bulut \\ Department of Otorhinolaryngology, Corlu Private Reyap Hospital, Istanbul Rumeli University, Istanbul, Turkey
}

\begin{abstract}
Background In lower lateral cartilage (LLC) surgery, cephalic trimming poses risks for the collapse of the internal and external nasal valves, pinched nose, and drooping deformity. The cephalic lateral crural advancement (CLCA) technique presented herein was aimed at using a flap to increase nasal tip rotation and support the lateral crus, in addition to the internal and external nasal valves, by avoiding grafts without performing excision.

Methods This study included 32 patients ( 18 female and 14 male) and the follow-up period for patients having undergone primer open rhinoplasty was 12 months. The LLC was elevated from the vestibular skin using the CLCA flap. A cephalic incision was performed without cephalic trimming. Two independent flaps were formed while preserving the scroll ligament complex. The CLCA flap was advanced onto the lower lateral crus while leaving the scroll area intact. The obtained data were analyzed retrospectively.

Results The mean age of the patients was 31.6 years (range, $20-51$ years). The Rhinoplasty Outcome Examination scores after 12 months varied from 90 to100 points, and 93\% of patients reported perfect satisfaction. At a 1-year follow-up, the patients' nasal patency (visual analogue scale) rose from $4.56 \pm 1.53$ (out of 10) to $9.0 \pm 0.65(P<0.001$ ).

Conclusions The CLCA flap led to better nasal tip definition by protecting the scroll area, increasing tip rotation, and supporting the internal and external nasal valves without cephalic excision.
\end{abstract}

\section{Keywords Rhinoplasty / Aesthetic / Ligaments / Rotation / Surgical flaps}

Received: August 21, 2020 • Revised: January 12, 2021 • Accepted: January 28, 2021

pISSN: 2234-6163 • elSSN: 2234-6171 • https://doi.org/10.5999/aps.2020.01648• Arch Plast Surg 2021;48:158-164

\author{
Correspondence: Fuat Bulut \\ Department of Otorhinolaryngology, \\ Corlu Private Reyap Hospital, Istanbul \\ Rumeli University, Mehmet Silivrili \\ Street Number: 38 , Silivri, Istanbul \\ 34570, Turkey \\ Tel: +90-212-866-01-01 \\ Fax: +90-212-866-01-25 \\ E-mail: bulutfuat40@yahoo.com
}

\section{INTRODUCTION}

Nasal tip surgery is pivotal in rhinoplasty, and a large part of this process consists of interventions on the lower lateral cartilage (LLC). The LLC, also known as the major alar cartilage, is a pair of $\mathrm{V}$-shaped cartilages supporting the medial and lateral walls of the nostril and forming the dome of the nasal tip.

The trimming of the cephalic part of the LLC alters the anatomy of the alar part of the nose. Cephalic trimming of the LLC may cause long-term sequelae and many potential complications, which are divided into functional and aesthetic issues: functional issues include collapse of the valve, while aesthetic is- sues include alar retraction, nasal tip asymmetry, a pinched tip, and the loss of aesthetic projection [1].

Deformities of the LLC may lead to functional and aesthetic handicaps; therefore, its width and length are critical for the outcomes of nasal tip surgery. Typically, the mean horizontal length of the lateral crus is $12 \mathrm{~mm}$, and the vertical width is half of its length-about $6 \mathrm{~mm}$ [2]. To have better aesthetics of the nasal tip and preserve the supporting function of the LLC, a thickness of the LLC of at least $0.7 \mathrm{~mm}$ was suggested [3]. Furthermore, connections of the LLC with the upper lateral cartilage (ULC) through ligaments and attachments to the adjacent soft tissue, such as the skin, are crucial. The cephalic part of the lateral crus 
of the LLC plays a significant role in nasal tip deformation, such as bulbous nasal tip formation, boxy or pinched nasal tip deformity, and drooping nasal tip [4].

There are many manoeuvres to increase nasal tip projection and reshape a wide tip. These manoeuvres include sutures, grafts, and flaps. Currently, cephalic trimming is frequently preferred by surgeons. The main limiting factor of cephalic trimming is the formation of dead spaces, which in the long term may cause uncontrolled scarring during recovery and an unnatural nasal tip definition. Cartilage grafts are effective in many cases, such as those involving weak or concave lower lateral crus, but they also pose risks, such as resorption, malposition, or distortion of the LLC or palpation of cartilage by patients $[5,6]$.

In nasal tip surgery, the cephalic lateral crural advancement (CLCA) flap narrows the tip and strengthens both the internal and external nasal valves. It also increases tip rotation, supports the lateral nasal wall, and allows medialization of the tip-defining points. Among the procedures on the LLC, the CLCA flap, which is a manoeuvre protecting the three-dimensional structure of the nose can result in more aesthetically favorable and natural-looking nose outcomes without performing excisions and using cartilage grafts.

\section{METHODS}

\section{Patient selection}

This study consisted of 32 ( 18 female and 14 male) patients. The mean age of the patients was 31.6 years (range, 20-51 years). Two patients were excluded from the study because they objected to sharing their medical information. The patients' follow-up period was 12 months. The study only included patients who had undergone primer open rhinoplasty. During the study, the CLCA flap was not used on patients who did not need nasal tip surgery. Those who demanded revision nasal tip surgery were also excluded from the study. Indications for performing this manoeuvre included a weak LLC, reduced volume of the LLC, a bulbous or boxy nasal tip, weakness of the internal and external nasal valves, and concavity of the LLC. The study was performed on human subjects for biomedical research in line with the Declaration of Helsinki. Before the operation, all patients were provided with extensive information about the study. Preoperatively, all patients were well informed about the study and provided written informed consent to undergo the operation and for their photographs to be published for educational purposes. All operations were performed by a single surgeon (FB). After the operation, all the patients were photographed from six different angles at 1, 3, 6, and 12 months using a digital camera, and video-recorded. The nasal tip projection, tip angle, dorsum-supratip relation, supratip-tip relation, tipcolumella relation, and columella-nasolabium relation were evaluated from photographs. Patient outcomes were evaluated with the Rhinoplasty Outcome Examination (ROE) [7] before surgery and at their 1-year follow-up appointment. The ROE survey is an objective tool designed to examine results and patient satisfaction after rhinoplasty. Patient responses were calculated as a score ranging from 0 to 4 points for each question. The patients were asked six questions, and a score of 4 points was considered the best. The total score was multiplied by 100 after dividing by 24 . A score greater than 95 was accepted as a perfect outcome and indicated high patient satisfaction. The patients were also studied in terms of functionality. A visual analogue scale or nasal opening score is also a simple and reliable method to assess nasal obstruction [8]. Functional improvement was assessed with the self-evaluation of nasal patency, ranging from 0 (complete obstruction) to 10 (optimal airflow).

\section{Surgical technique}

All patients in this study underwent surgery under general anesthesia. A local anesthetic was administered with $1 / 100,000$ adrenaline solution infiltration into the incision sites before the skin incisions were made to prevent excessive bleeding. Rhinoplasty in the selected cases such as bulbous, drooping nasal tips or cephalic malposition of the LLC started with a vestibular incision. In all patients, a transcolumellar inverted-V incision was made using a no. 15 scalpel blade. Then, the operation continued with forming a columellar flap. After the infra-cartilaginous incision, we proceeded with the subperichondrial dissection. A drawing of the CLCA flap is demonstrated in Fig. 1 and the actual surgical procedure of the CLCA flap is shown in Fig. 2. The LLC was lifted with $70^{\circ}$ sharp scissors from the inner surface of the nasal mucosa to $3-4 \mathrm{~mm}$ from the accessory cartilage area and $2-3 \mathrm{~mm}$ closer to the ULC junction. Then, an incision was made parallel to the caudal part of the lateral crus before cephalic trimming. A cephalic flap was created by leaving a width of 6 $\mathrm{mm}$ in the lower lateral crus. As a result of these steps, two different flaps were created, the lateral crus pivot flap and the superiorly located cephalic flap. The created pivot flap-shaped CLCA flap was advanced under the cephalic flap. It was detected with two or three 5.0 polydioxanone to fix the flaps to each other and to the vestibule skin.

After cephalic incision of the LLC and advancement of the cephalic part of the LLC lateral to the caudal part, this technique both improved nasal tip definition but also increased tip rotation.

Thus, extremely convex or concave lateral crura were reconstructed without using additional sutures or cartilage grafts. 
(1)

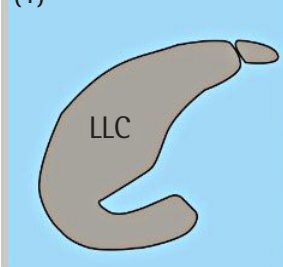

(2)

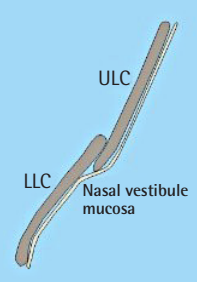

(3)

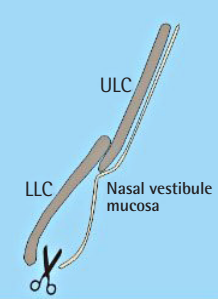

(4)

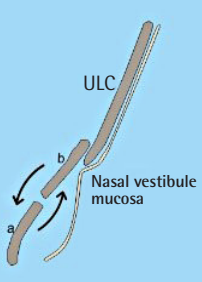

(5)

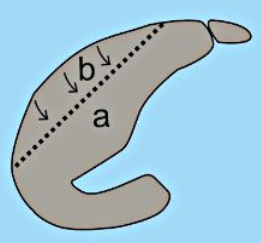

(6)

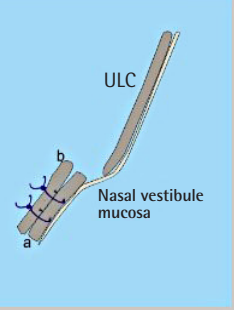

Fig. 1. Schematic drawing of nasal tip surgery technique. (1) Normal anatomy of the lower lateral cartilage (LLC). (2) Dissection of the LLC from the vestibular skin to $2 \mathrm{~mm}$ closer to its junction with the upper lateral cartilage (ULC). (3) Incision of the LLC to create a cephalic flap (Note that the scroll area is left intact to protect the caudal end of the ULC). $(4,5)$ Advancement of the cephalic flap over the lower lateral crus. (6) Suturing the cephalic advancement flap lateral to the caudal portion of the LLC with two or three 5-0 polydioxanone sutures.
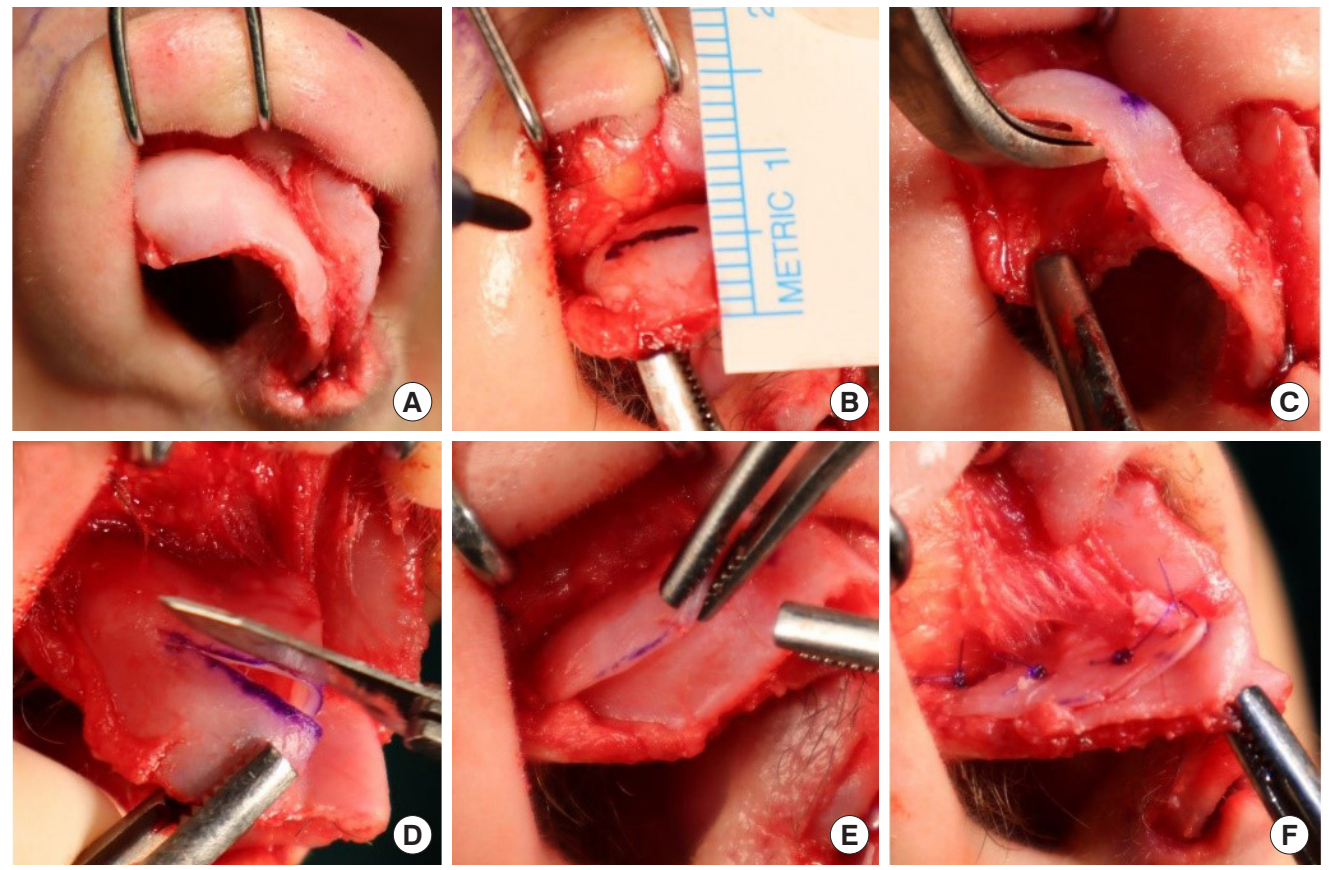

Fig. 2. Surgical procedure for the CLCA flap. (A) Normal anatomy of the lower lateral cartilage (LLC). (B) Dissection of the LLC from the vestibular skin to $2 \mathrm{~mm}$ closer to its junction with the upper lateral cartilage (ULC). (C) Incision of the LLC to create a cephalic flap (Note that the scroll area was left intact to protect the caudal end of the ULC). (D) Advancement of the cephalic flap over the lower lateral crus. (E) The created pivot flap-shaped cephalic lateral crural advancement (CLCA) flap is advanced under the cephalic flap. (F) It is detected with two or three 5.0 PDS to fix the flaps to each other and to the vestibule skin.

Furthermore, excisions were no longer required to shorten the lower lateral crura. None of the patients in this study required resection of the cephalic part of the LLC or additional sutures or cartilage grafts for lateral crural repair.

\section{RESULTS}

A total of 32 patients ( 18 female and 14 male) completed the 12-month follow-up period. Patient photographs were evaluated by the surgeon with preoperatively nasal opening score and after a 12-month follow-up period. The ROE scores after 12 months varied between 90 and 100 points, and perfect satisfaction was found for $93 \%$ of patients. Data were expressed as mean \pm standard error of the mean. After comparing the preoperative and postoperative photos, the first surgeon gave 2 points to 26 patients and 1 point to the remaining patients. The second surgeon gave 2 points to 28 patients and 1 point to the remaining patients; none of them gave 0 points to any patients. Interrater reliability in the analysis of aesthetic results showed significant agreement (Fleiss kappa $=0.765 ; \mathrm{P}<0.001$ ).

Functional outcomes were also analyzed. After a 12-month follow-up period, the patients' nasal opening scores rose from $4.56 \pm 1.53$ (out of 10 ) to $9.0 \pm 0.65$ (Wilcoxon signed-rank test; $\mathrm{P}<0.001)$.

In 17 patients, convexity was observed in the LLC, and in 15 patients, there was concavity in the LLC before the operation. 

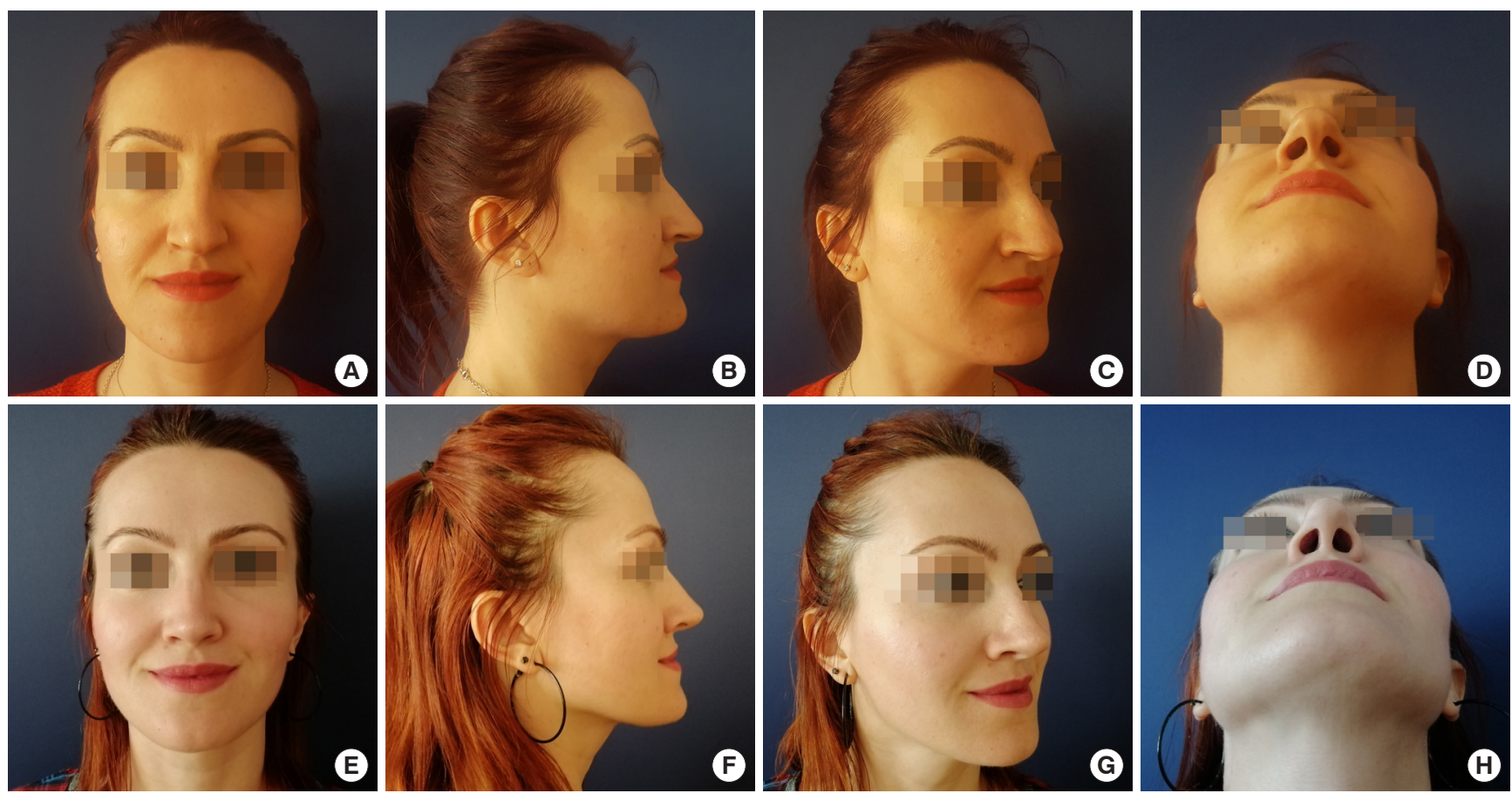

Fig. 3. Clinical photographs of the patient. Preoperative (A) frontal, (B) right lateral, (C) oblique, and (D) worm's eye images of a 32-year-old female patient. Postoperative (E) frontal, (F) right lateral, (G) oblique, and (H) worm's eye images taken 14 months after the operation. The patient's nasal form was thin-skinned and drooping, and the nasal wings were weak. Prior to the operation, the nasal opening score was 6 out of 10. The nose was deviated to the left in the anterior portion and deviated to the right in the posterior portion. The bilateral lower conchae were hypertrophic. The surgical procedure followed a protocol of open-technique transcolumellar inverse-V incision. The septum was excised from the anterior by $0.3-\mathrm{cm}$ cartilage, bilateral lateral osteotomy was performed and the nose hump was trimmed for 30 seconds. A bilateral spreader graft was placed and a bilateral cephalic lateral crural advancement flap was formed. The scroll area was protected. A 0.4-cm lateral crural steal suture was extracted. A new dome point could thus be identified. Dome sutures were applied. The interdomal suture was extracted. A strut graft was placed. After suturing, a nasal silicone splint plaster was placed into the bilateral Doyle splints. Upon applying a SteriStrip and cast, the operation was ended.

They all recovered with perfect outcomes. In all patients, the LLC was $12 \mathrm{~mm}$ or larger, and the CLCA flap was fixed on the lateral surface of the remaining LLC. The patients had no functional complaints after the operation. The same surgeon (FB) performed all procedures between September 2018 and June 2019. The follow-up period was between 12 and 18 months (median, 14.8 months). The aesthetic and functional results were satisfactory for both patients and surgeons (Fig. 3).

\section{DISCUSSION}

This study aimed to investigate the effects of dissecting the LLC from the vestibular skin and mucosa and using a CLCA flap without performing cephalic trimming of nasal tip rotation. Using this manoeuvre, we also reconstructed bulbous nasal tips and supported both the internal and external nasal valves while preserving the scroll area.

Nasal tip surgery is a challenging and complicated part of rhinoplasty. The major component of nasal tip surgery formation of an aesthetic LLC. In LLC surgery, each cephalic trimming manoeuvre can-in the long term-pose possible risks for pinched nasal tip deformity, loss of tip rotation, collapse of the internal and external nasal valves, unnatural and aesthetically unfavorable results in the supra-alar area, and weakness and deformities in the alar rim. Thus, to narrow the nasal tip, we should not perform radical excision because manoeuvres involving cephalic trimming excisions could lead to the formation of dead spaces around the surgical field. In the long term, these dead spaces may cause re-draping of the skin, which may lead to unnatural nasal tip definition (e.g., undesired shadows in the supraalar crease) and sometimes a decrease in tip rotation. Surgical techniques that would recover the three-dimensional anatomy of the nasal alar and tip regions would advance protective nasal tip surgery as well. With our CLCA flap technique, one can support the alar rim and prevent drooping of the nasal tip and the formation of dead spaces.

Nasal tip size, shape, and position are determined by the length, width, and strength of the lateral crus. It has been reported that a nose with concave lateral crus and convex domal segment of the middle crus provides a more aesthetic domal junc- 
tion and domal outline [9]. In nasal tip surgery, the position and shape of the lateral crus play a major role in terms of functionality and aesthetics. The lateral crus plays an important role in the appearance of the borders of the alar rim. Functionally, the LLC is an important component of the external nasal valve, which forms the first section of the nasal airway and supports the internal nasal valve. Preservation of the ligament complex in the scroll area of the lateral crus that connects the LLC to the ULC is a vitally imperative consideration in nasal tip surgery. Our CLCA flap technique was able to increase the strength of the LLC by keeping the scroll ligament complex intact.

Toriumi and Checcone [4] emphasized the necessity of understanding the harmony between the external nasal contour and the components under the tip. Their statement underscores the significance of preserving the contours of the lower lateral crura. If they are not adequately preserved, it is likely there would be a pinched nasal tip, and in some extreme cephalic trimming cases, intervention may lead to alar collapse. Therefore, for many patients, a preventative alar batten graft medial to the supra-alar line is advised to avert the collapse of the lateral nasal wall after cephalic trimming. Our CLCA flap technique does not employ any cephalic trimming, so we did not require a cartilage graft to support the lateral nasal wall.

Gruber et al. [10] emphasized the importance of preserving the lateral crura and cephalic part of the LLC in surgery sparing with LLC and its cephalic portion. In modern rhinoplasty, the role of cephalic trimming of the LLC is questionable in terms of its long-term outcomes [11]. Cephalic trimming damages the scroll area, and in the long term, it may cause unsuccessful recovery of the scroll area, formation of scars, loss of support on the cartilage and ligament junction, and adverse results over the nasal tip area [12].

Sheen [13] first described the cephalic malposition of the LLC as bulbosity, boxy nasal tip deformation, and parenthesisshaped tip deformity from the frontal view. In general, cephalic malposition results in supratip swelling and a bulbous nasal tip. Furthermore, it may frequently cause loss of alar support, resulting in external nasal valve collapse, which may lead to alar retraction. Cephalic malposition of LLCs may result in alignment of the nasal tip with the medial canthus rather than the lateral canthus. The anatomical angle of the LLC to the midline must be $45^{\circ}$, but cephalic malposition decreases the angle to $30^{\circ}$. Cephalic trimming is not a physiological manoeuvre for repositioning the LLC in primer rhinoplasty. It has been reported that repositioning of bulbous and cephalic malposition of the lateral crus can be achieved by cephalic trimming, dissecting from the vestibular skin, and adding a strut graft below the lateral crura [14]. Our CLCA flap technique allows lateral crural rotation in patients with cephalic malposition. This technique achieves better nasal tip definition without performing additional excisions and cartilage grafts.

The lateral crural spanning suture technique has been suggested to repair the convexity of the lateral crura [15]. In patients with weak cartilage, dome sutures may progress to pinched nasal tip deformity because of the disturbance of the normal lateral crural convexity. The tightness of dome sutures and rigid crura promotes pinched nasal tip formation.

The CLCA flap is a manoeuvre to be used in patients with lateral crural convexity. The lateral crural mattress suture technique has also been defined for the correction of lateral crural concavity. Studies have suggested that cephalic trimming and dissection of the LLC from the vestibular skin can be applied to bulbous and cephalic malpositioning of the lateral crura [16].

Dome sutures decrease the concavity deformation of lateral cartilage in most of the concave LLC cases, but these sutures lead to the displacement of the caudal border of the lateral crus. The cephalic part of the lateral crus can be pulled inferiorly, but sometimes using just a few sutures may be inadequate. Furthermore, it may be compulsory to apply a lower lateral crural strut graft. Both in the short and long terms, these manoeuvres mostly end up as pinched nasal tip deformity.

The CLCA flap technique enables crural rotation and avoids such problems in extreme concavity or convexity and cephalic misposition of the lower lateral crura, without demanding additional sutures or cartilage grafts. If there is wide lower lateral crus on the vertical plane, the CLCA flap can be adopted. Even if wide lower lateral crura seem oriented normally in the anatomical position, some supratip fullness would still be present on the lateral view. Although cephalic trimming lessens supratip fullness and the width of the LLC in the vertical plane initially, it may lead to poor nasal tip definition in the long term.

There should not be excessive intrusion on the LLC in thinskinned patients because LLCs may lose their strength, thereby making the lateral nasal walls weak. This could lead to the emergence of deformations of the alar rim. Studies have reported that disproportionate resection of the lateral crura to achieve better nasal tip definition, especially in thick-skinned patients, causes multiple complications. Some of these complications include tip asymmetry, external nasal valve failure, alar failure, drooping nasal tip, and tip definition loss [17]. Weak lower lateral crura cause both adverse functional and aesthetic outcomes. Studies have suggested that weak lateral crus of the alar cartilage may evolve into alar rim concavity and even alar rim (external nasal valve) collapse [18]. When applied to thin and weak lateral crura, the CLCA flap supports the lower lateral crus by advancing the cephalic flap over the LLC. 
Thanks to recent cadaveric studies, the relationship between the lower lateral crura and the surrounding tissues has been better understood. A study of turn-in folding of the cephalic portion of the lateral crus with weak and ineffective alar parts emphasized the importance of not trimming the cephalic part of the LLC [19]. After that, the cephalic turn-in-the-flap technique was defined [20]. These two techniques were later modified [21]. A study described the superior-based transposition flap as not involving cephalic excision, and fixing the flap harvested from the cephalic part of the LLC over the rest of the LLC [22]. The difference of our CLCA flap technique from the superiorbased transposition flap is that in addition to the cephalic part of the LLC, the lateral crus is also separated from the vestibular skin. The advantage of the CLCA flap technique is that it allows rotation of the lateral crus in the cephalic direction together with the cephalic flap. Therefore, the CLCA flap contributes to cephalic rotation of the nasal tip. This manoeuvre also allows the lateral crus flap and cephalic flap to be clamped on each other effortlessly.

The scroll ligament complex that acts as a gliding joint between the LLC and the ULC has been verified in recent cadaveric studies. This complex is mainly formed by ligaments and cartilages, but it also contains a deep layer of the superficial musculoaponeurotic system and perichondrium [23]. This arrangement supports both the internal and external nasal valves by securing the unity of the ULC and LLC. Our CLCA flap technique protects the scroll ligament complex, allowing a gliding action between the LLC and the ULC. It enables three-dimensional control by preserving the scroll ligament complex in the nasal tip.

The CLCA flap, also known as the preservation of the nasal tip manoeuvre, has proved to be an important technique in rhinoplasty. It is an effective and controlled manoeuvre to position the lateral crura. This study presented several advantages of the CLCA flap. In summary: (1) the LLC can be strengthened; (2) it is possible to eliminate extreme concavity or convexity of the LLC without using a cartilage graft and extra sutures; (3) nasal tip rotation improves; (4) the CLCA flap provides aesthetic wing contours; (5) the scroll area is preserved; (6) the internal and external nasal valves are supported; or (7) nasal tip definition improves.

In conclusion, the CLCA flap technique preserves the connection between cartilages, representing the functional anatomy and supporting the internal and external valves without cartilage excision. We believe that this technique is a valuable modern preservation rhinoplasty technique. By applying the CLCA flap technique in nasal tip surgery for suitable candidates, we hope to achieve more natural, aesthetic, and functional results. The main limitation of this study is that operations were performed by one surgeon only, with a limited number of patients, and a longer follow-up period would be required. Further studies with a higher number of cases and longer follow-up periods would provide a better understanding of the efficiency of the CLCA flap technique.

\section{NOTES}

\section{Conflict of interest}

No potential conflict of interest relevant to this article was reported.

\section{Ethical approval}

The study was approved by the Institutional Review Board of Tekirdag Namık Kemal University Faculty of Medicine (IRB No. 2020.275.12.20) and performed in accordance with the principles of the Declaration of Helsinki. Written informed consent was obtained.

\section{Patient consent}

The patients provided written informed consent for the publication and the use of their images.

\section{ORCID}

Fuat Bulut

https://orcid.org/0000-0003-3803-7987

\section{REFERENCES}

1. Nagarkar P, Stark RY, Pezeshk RA, et al. Role of the cephalic trim in modern rhinoplasty. Plast Reconstr Surg 2016;137: 89-96.

2. Patel JC, Fletcher JW, Singer D, et al. An anatomic and histologic analysis of the alar-facial crease and the lateral crus. Ann Plast Surg 2004;52:371-4.

3. Hatzis GP, Sherry SD, Hogan GM, et al. Observations of the marginal incision and lateral crura alar cartilage asymmetry in rhinoplasty: a fixed cadaver study. Oral Surg Oral Med Oral Pathol Oral Radiol Endod 2004;97:432-7.

4. Toriumi DM, Checcone MA. New concepts in nasal tip contouring. Facial Plast Surg Clin North Am 2009; 17:5590.

5. Rohrich RJ, Raniere J Jr, Ha RY. The alar contour graft: correction and prevention of alar rim deformities in rhinoplasty. Plast Reconstr Surg 2002;109:2495-505.

6. Guyuron B. Alar rim deformities. Plast Reconstr Surg 2001; 107:856-63.

7. Alsarraf R. Outcomes research in facial plastic surgery: a re- 
view and new directions. Aesthetic Plast Surg 2000;24:1927.

8. Constantinides M, Galli SK, Miller PJ. A simple and reliable method of patient evaluation in the surgical treatment of nasal obstruction. Ear Nose Throat J 2002;81:734-7.

9. Daniel RK. The nasal tip: anatomy and aesthetics. Plast Reconstr Surg 1992;89:216-24.

10. Gruber RP, Zang A, Mohebali K. Preventing alar retraction by preservation of the lateral crus. Plast Reconstr Surg 2010; 126:581-8.

11. Sazgar AA, Amali A, Peyvasty MN. Value of cephalic part of lateral crus in functional rhinoplasty. Eur Arch Otorhinolaryngol 2016;273:4053-59.

12. Davis RE. Lateral crural tensioning for refinement of the wide and underprojected nasal tip: rethinking the lateral crural steal. Facial Plast Surg Clin North Am 2015;23:23-53.

13. Sheen JH. Rhinoplasty: personal evolution and milestones. Plast Reconstr Surg 2000; 105:1820-52.

14. Gunter JP, Friedman RM. Lateral crural strut graft: technique and clinical applications in rhinoplasty. Plast Reconstr Surg 1997;99:943-52.

15. Tebbetts JB. Shaping and positioning the nasal tip without structural disruption: a new, systematic approach. Plast Reconstr Surg 1994;94:61-77.
16. Gruber RP, Nahai F, Bogdan MA, et al. Changing the convexity and concavity of nasal cartilages and cartilage grafts with horizontal mattress sutures: part II. clinical results. Plast Reconstr Surg 2005;115:595-606.

17. Gubisch W, Eichhorn-Sens J. Overresection of the lower lateral cartilages: a common conceptual mistake with functional and aesthetic consequences. Aesthetic Plast Surg 2009;33:6-13.

18. Gunter JP, Rohrich RJ. Correction of the pinched nasal tip with alar spreader grafts. Plast Reconstr Surg 1992;90:8219.

19. Tellioglu AT, Cimen K. Turn-in folding of the cephalic portion of the lateral crus to support the alar rim in rhinoplasty. Aesthetic Plast Surg 2007;31:306-10.

20. Murakami CS, Barrera JE, Most SP. Preserving structural integrity of the alar cartilage in aesthetic rhinoplasty using a cephalic turn-in flap. Arch Facial Plast Surg 2009;11:126-8.

21. Apaydin F. Lateral crural turn-in flap in functional rhinoplasty. Arch Facial Plast Surg 2012;14:93-6.

22. Tas S. Superior-based transposition flap: a novel technique in rhinoplasty. Aesthet Surg J 2019;39:720-32.

23. Daniel RK, Palhazi P. The nasal ligaments and tip support in rhinoplasty: an anatomical study. Aesthet Surg J 2018;38: $357-68$. 